\title{
Cortical Microvascularization and Leptomeningeal Collaterals in Moyamoya Disease
}

\author{
Shunji Mugikura ${ }^{a}$ Miki Fujimura $^{\mathrm{b}}$ Shoki Takahashi ${ }^{\mathrm{a}}$ \\ Departments of a Diagnostic Radiology, and beurosurgery, Graduate School of Medicine, Tohoku University, Sendai, \\ Japan
}

Dear Sir,

We read with great interest the article by Kim et al. entitled 'Neovascularization precedes occlusion in moyamoya disease: angiographic findings in 172 pediatric patients' [1]. This study focused on cortical microvascularization (CM), which was originally described as being visualized on indocyanine green (ICG) video angiograms under surgical microscopy (microscopic CM) by Czabanka et al., who found that microscopic CM in moyamoya disease was characterized by significantly increased microvascular density and microvascular diameter, leading to increased microvascular surfaces. They concluded that the increased microscopic CM specifically found in this disease refers to the cortical vessels being maximally dilated to compensate for impaired cerebral blood flow [2].

Meanwhile, Kim et al. investigated the presence of $\mathrm{CM}$ using conventional angiography (angiographic CM) in relation to disease severity on angiograms (Suzuki stage) $[3,4]$ in 172 pediatric patients with moyamoya disease. Angiographic CM was defined as enlarged and winding distal cortical arteries seen in the arterial phase on conventional angiograms and categorized into anterior or posterior CM depending on source: anterior $\mathrm{CM}$ from the internal carotid artery (ICA) system including the anterior (ACA) or middle (MCA) cerebral arteries, and posterior CM from the poste- rior cerebral artery (PCA). They found that angiographic CM was frequently observed even at early Suzuki stages, which further developed thereafter but gradually decreased with advancing Suzuki stages. In addition, about two-thirds of hemispheres with normal antegrade MCA flow on angiography and normal perfusion status on single-photon emission computed tomography contained angiographic CM.

Although Kim et al. did not discuss whether the presence of angiographic CM actually represented increased microscopic $\mathrm{CM}$, we suggest that angiographic CM could represent the development of leptomeningeal collateral arteries (LCAs) rather than an increase in microscopic CM for the following three reasons.

First, it seems that angiographic CM in the study of Kim et al. and the LCAs described in the literature including our study [4] share common temporally sequential changes in frequency according to Suzuki stages. From the sequential viewpoint in moyamoya disease, steno-occlusive changes initially involve the ICA system, followed by the PCA $[5,6]$. In hemispheres with Suzuki stage 1 showing mild stenotic ICA without basal collaterals, LCAs could develop between the ACA and MCA branches (anterior LCAs) with some LCAs being well developed (good or moderate) from the PCA (posterior LCAs) (posterior LCAs: two of six hemispheres (33\%) with
Suzuki stage 1 in our study [6]). In this stage, the posterior CM was found in about $20 \%$ in the study of Kim et al.

Anterior LCAs could increase in stage 2, which shows more stenotic ACAs or MCAs with intensified basal collaterals, but not enough to compensate for the ischemia present because of proximal stenosis, while posterior LCAs might increasingly develop from the PCA without stenosis (posterior LCAs: 15/19 (79\%) hemispheres of patients with Suzuki stage 2 in our study [6]). Posterior CM was found in this stage in about $70 \%$ in the study of Kim et al.

In stages 3-4, which show more advanced stenosis or occlusion of the ACA and MCA with well-developed basal collaterals, anterior LCAs could drastically decrease, whereas posterior LCAs from the less stenotic PCA could be well preserved to compensate for ischemia in the anterior circulation (posterior LCAs: 60/93 (68\%) hemispheres with Suzuki stages 3-4 in our study). In these stages, posterior CM was found in about $70-80 \%$ in the study of Kim et al.

In stages 5-6, during which anterior LCAs and basal collaterals almost disappear, only the posterior LCAs could remain (posterior LCAs: 4/19 (21\%) hemispheres with Suzuki stages 5-6 in our study). In these stages, posterior CM was found in about $60 \%$ in the study of Kim et al. Since posterior LCAs decreased as PCA lesions
KARGER 125

(c) 2015 S. Karger AG, Base

0014-3022/15/0736-0351\$39.50/0
Shunji Mugikura, MD, $\mathrm{PhD}$

Department of Diagnostic Radiology

Graduate School of Medicine, Tohoku University

1-1 Seiryo-machi, Aoba-ku, Sendai, 980-8574 (Japan)

www.karger.com/ene 
advanced, the relative decrease in the posterior LCAs through stages 5-6 was ascribed to frequent steno-occlusive involvement of the PCA in later Suzuki stages as shown in our study [6]. Therefore, the differential prevalence of the posterior LCA in stages 5-6 between our study (21\%) and that of Kim et al. (60\%) presumably reflected the higher prevalence of PCA involvement $(95 \%)$ in our study, although prevalence was not given by Kim et al.

Considering the common sequential change in frequency, we suspect that angiographic CM could represent a form of LCAs. If that is the case, it is no wonder why angiographic CM may occur in the early stage before significant hemodynamic impairment as found by Kim et al. because LCAs (or angiographic CM) are known to possibly develop in the early Suzuki stages [6].

Differences in the observation methods and definitions are another reason to sus- pect that angiographic CM differs from microscopic CM. Microscopic CM as described by Czabanka et al. included both arterioles and venules with an average diameter of $0.24 \pm 0.03 \mathrm{~mm}$ observed by ICG video angiograms under surgical microscopy [2]. On the other hand, angiographic CM was defined by Kim et al. and observed based on the arterial phase on conventional catheter angiograms, which should have been capable of evaluating relatively larger cortical arteries encompassing leptomeningeal anastomoses [7] but did not enable evaluation of microscopic CM. Thus, we suspect that the two CM types (angiographic and microscopic) are not identical.

Another possible explanation for the difference between angiographic and microscopic CM is that the increased microscopic CM is associated with decreased cerebrovascular reserve capacity [8], while the presence of angiographic CM was independent of whether cerebrovascular re- serve was decreased in the study of Kim et al. Although both angiographic CM and microscopic CM may develop in the presence of ischemia in moyamoya disease, we believe that they represent different compensating effects: microscopic $\mathrm{CM}$, maximally dilated cortical vessels with reduced vascular resistance, would contribute to preserving cerebral blood flow, whereas angiographic CM would involve relatively larger LCAs that work as collateral vessels [9].

\section{Statement of Ethics}

We declare that this manuscript does not contain clinical studies or patient data.

\section{Disclosure Statement}

We have no conflict of interests.

\section{References}

1 Kim SJ, Son TO, Kim KH, et al: Neovascularization precedes occlusion in moyamoya disease: angiographic findings in 172 pediatric patients. Eur Neurol 2014;72:299 305.

-2 Czabanka M, Peña-Tapia P, Schubert GA, Woitzik J, Vajkoczy P, Schmiedek P: Characterization of cortical microvascularization in adult moyamoya disease. Stroke 2008;39: 1703-1709.

-3 Suzuki J, Takaku A: Cerebrovascular 'moyamoya' disease. Disease showing abnormal net-like vessels in base of brain. Arch Neurol 1969;20:288-299.
4 Fujimura M, Tominaga T: Lessons learned from moyamoya disease: outcome of direct/ indirect revascularization surgery for 150 affected hemispheres. Neurol Med Chir (Tokyo) 2012;52:327-332.

5 Mugikura S, Takahashi S: Letters to the editor: posterior cerebral artery involvement and pediatric moyamoya diseaes. J Neurosurg Pediatr 2014;14:434-435.

6 Mugikura S, Takahashi S, Higano S, et al: The relationship between cerebral infarction and angiographic characteristics in childhood moyamoya disease. AJNR Am J Neuroradiol 1999;20:336-343.
7 Brozici M, van der Zwan A, Hillen B: Anatomy and functionality of leptomeningeal anastomoses: a review. Stroke 2003;34:27502762.

8 Czabanka M, Peña-Tapia P, Schubert GA, et al: Clinical implications of cortical microvasculature in adult Moyamoya disease. J Cereb Blood Flow Metab 2009;29:1383-1387.

-9 Mori N, Mugikura S, Higano S, et al: The leptomeningeal 'ivy sign' on fluid-attenuated inversion recovery MR imaging in moyamoya disease: a sign of decreased cerebral vascular reserve? AJNR Am J Neuroradiol 2009;30: 930-935. 